\title{
3 Research Suare \\ Relationship Between Sedentary Behavior And Sleep Indicators In Patients With Chronic Schizophrenia
}

\section{Rei Takenouchi}

College of Nursing Art and Science, University of Hyogo

Masashi Shibata

College of Nursing Art and Science, University of Hyogo

Ikuko Kishida

Fujisawa Hospital

Chie Ishii

Fujisawa Hospital

Norio Ishii

Fujisawa Hospital

Miwa Kawada

College of Nursing Art and Science, University of Hyogo

Momoko Buyo ( $\nabla$ momo-bu@sahs.med.osaka-u.ac.jp )

Osaka University Graduate School of Medicine

\section{Research Article}

Keywords: sedentary, insomnia, schizophrenia, sleep indicators, sleep

Posted Date: January 17th, 2022

DOI: https://doi.org/10.21203/rs.3.rs-1212813/v1

License: (c) (i) This work is licensed under a Creative Commons Attribution 4.0 International License. Read Full License 


\section{Abstract}

Background: To investigate the relationship between daytime sedentary behavior and physical activity and sleep in patients with chronic schizophrenia, particularly focusing on insomnia symptoms, a hallmark of insomnia in patients with schizophrenia.

Method: The subjects were 20 patients with chronic schizophrenia more than 1 year after onset (12 men and 8 women with a mean age of $59.0 \pm 9.8$ years), of whom 4 were outpatients and 16 were inpatients. Sedentary behavior performance and physical activity index (Total sedentary behavior time and sedentary behavior bout number, low-intensity physical activity, MVPA, and steps) were assessed by wearing a triaxial accelerometer (GT3X-BT). Total bed time (TIB), total sleep time (TST), sleep latency (SIL), wake after sleep (WASO), and sleep efficiency (SE) were calculated using a small body dynamometer as an objective sleep index. Both the triaxial accelerometer and the small body dynamometer were worn for 1 week, and the average value of each index was calculated.

Results: Total sedentary behavior time was significantly positively associated with TIB and SL $(r=.499, p$ $<.05 ; r=.543, p<.05)$. Sedentary behavior bout number was negatively significantly associated with SE $(r=-.592, p<.01)$ and positively associated with WASO $(r=.503, p<.01)$. MVPA was significantly positively associated with $S E(r=.536, p<.05)$ and negatively associated with WASO $(r=-.58, p<.01)$.

Conclusion: Daytime sedentary behavior in patients with chronic schizophrenia was associated with insomnia symptoms. It was suggested that decreasing their sedentary behavior time and increasing moderate-to-vigorous intensity activities may improve their sleep quality.

In this study, though the relation has not been recognized in CP conversion value and sedentary behavior position action index, the relation between antipsychotic dose and total sedentary behavioral time needs further investigation.

\section{Q. Introduction}

Many patients with schizophrenia have insomnia symptoms, and it is difficult to improve the symptoms only by pharmacotherapy. Approximately $80 \%$ of patients with schizophrenia have sleep disorders (Laskemoen et al., 2019), and studies comparing sleep in healthy subjects with that in schizophrenic patients without drug therapy suggest that insomnia is an essential feature of schizophrenia, with shorter total sleep time, longer sleep latency, and less sleep efficiency in schizophrenic patients than in healthy subjects (Chouinard, 2004). Main treatment methods of integration incontinence are pharmacotherapy and psychiatry rehabilitation, and the center of pharmacotherapy is the antipsychotic drug. Antipsychotics are effective not only for the symptoms of schizophrenia but also for insomnia. It has been reported that oral administration of clozapine and olanzapine increased total sleep time and shortened sleep latency (Kluge et al., 2014). However, schizophrenia often recurs due to discontinuation of medication, and it is said that the quality of sleep decreases when antipsychotic treatment is discontinued (Cohrs, 2008); therefore, it is difficult to expect improvement of insomnia in patients only 
with medication. In addition, qualitative studies interviewing patients with schizophrenia from Faulkner and Bee (2017) reported that patients with schizophrenia were aware of changes in their sleep after onset, and that they were aware that relying on improvised drugs was a temporary coping strategy that was not optimal. From these results, it is necessary to examine the method except for the ingestion for the improvement on insomnia of the integration incontinence patient.

Physical activity to reduce insomnia appropriate for patients with chronic schizophrenia should be considered. Exercise is said to improve the quality of sleep (sleep quality) in patients with psychiatric disorders, and it has been reported that continuing aerobic and resistance exercises, Tai Chi, and yoga for $6 \sim 12$ weeks is effective (Lenderman, et al., 2019). In the meantime, it is said that the activity quantity in the daytime lowers on the negative symptom which becomes remarkable in the integration incontinence patient in the chronic stage by losing the sociality and flattening the feeling. In addition, in Japan, there are many psychiatry sickbeds in comparison with foreign countries, and the average hospitalization days is long, and there are also many chronic stage patients who have been hospitalized over 1 year (OECD, 2020; Ministry of Health, Labour and Welfare, 2020). In addition, it seems to be difficult to continuously and independently carry out the motion (exercise), because the population ratio over 65 year-old in 2020 in Japan is $28.7 \%$ (Statistics Bureau of Japan, 2021), and the renewal in the past continues, and because the aging of the integration incontinence patient also advances.

By clarifying the relation between sedentary behavior behavior and sleep of the chronic stage integration incontinence patient, it is possible to examine the new intervention method to the sleep. Daytime physical activity is divided into sedentary behavior, low-intensity physical activity, and intermediate-intensity physical activity. Sedentary behavior behavior (Sedentary Behaviour) is defined as "All arousal behaviors with energy expenditure < 1.5 METs while seated, semi-recumbent, and recumbent" (Sedentary Behaviour Research Network, 2019). Moderate physical activity is defined as physical activity over 3 METs, and low intensity physical activity as physical activity of $1.5 \sim 3.0$ METs. Hospitalized patients with chronic schizophrenia have been reported to sit longer ( $9.6 \mathrm{~h}$ vs $5.6 \mathrm{~h})$ and take fewer steps $(6,628$ steps vs 10,976 steps) per day than healthy controls (Stubbs, et al., 2017a). And, the sedentary behavior hour in a day occupies $8.8 \mathrm{~h}$ and $58.7 \%$ of the awakening hour in chronic stage integration incontinence patients under ambulatory (Bueno-Antequera, et al., 2018).

On the relation between physical activity and sleep of the integration incontinence patient, the research which focused on the sedentary behavior behavior has seldom been observed. However, it is possible to examine the physical activity which can be continued more autonomously for improving the sleep by observing the relation between sleep and physical activity including not only physical activity strength but also sedentary behavior behavior.

The purpose of this study was to investigate the relationship between daytime sedentary behavior and physical activity and sleep in patients with chronic schizophrenia, especially focusing on insomnia symptoms, a hallmark of insomnia in patients with schizophrenia. In this study, it was decided that the 
sleep with problems such as sleep onset disorder, arousal in the middle, early morning arousal and sound sleep disorder was defined as insomnia.

\section{li. Research Methods}

\section{Target Audience}

The object satisfied all following four conditions. The 4 conditions were as follows: 1 ) Schizophrenia is diagnosed in DSM -5 and the disease name is recognized, 2) the description content on the research can be understood, 3) the filling in consent forms, etc. can be done, and 4) there is no physical functional problem on the mounting of the measuring instrument.

The selection of the object was carried out, when the patient who matched the condition was introduced from the ward manager of the research cooperation facilities, and when the researcher directly talked with the person, it was selected.

\section{Measurement Item}

Daily physical activity and insomnia symptom index were assessed by wearing measuring instruments for 1 week.

\section{1) Sedentary behavior behavior and physical activity indicators}

The subjects wore the ActiGraph GT3X-BT (ActiGraph, LLC, Pensacola, FL, USA) on their lumbar region for one week, excluding bathing time, from waking up in the morning to going to bed at night, and obtained physical activity for one week after equipment recovery using the memory function. The support person of the subject was asked to appropriately confirm whether the equipment can be fixed except for the bathing. From the obtained data, the mean values of total sedentary behavior time and sedentary behavior bout number, low intensity physical activity (light physical activity; LPA), moderate or more physical activity (moderate-to-vigorous physical activity; MVPA), and steps per day were calculated and adopted as quantitative indices of sedentary behavior behavior and physical activity. Total sedentary behavior time represents the total time of less than 1.5 METs of physical activity while awake, and the sedentary behavior bout number represents the total number of interruptions in sedentary behavior time of more than 5 minutes.

Low-intensity physical activity represents the total time of physical activity between 1.5 Ets and 3.0 METs, and MVPA represents the total time of physical activity over 3.0 METs.

The data of sedentary behavior action hour and low intensity activity hour, MVPA can know what kind of intensity activity the chronic phase integration incontinence patient carries out in the arousal hour except for the bed time. In addition, the effect of the interruption of the sedentary behavior action can be observed by the data of the sedentary behavior bout number. 


\section{2) Insomnia symptom index}

In this study, there was the correlation in the sleep polygraph, and it was made that the insomnia symptom index was objectively obtained using the actigraphy of the wrist watch type which is simply wearable. A small body dynamometer (AW-L, Mini-Mitter) was worn on the wrist of the non-dominant hand of the subject continuously for one week except for the bathing hour, and the rising and the rising time were recorded in the sleep diary. Using Actiware (Ver 5.57, Mini-Mitter), the following data on sleep variables were obtained from body movement data and time of waking and going to bed recorded in 1 min interval on the actigraph: total bed time (Time to rise from bed: TIB), total sleep time (The sum of the time spent in sleep among the total time spent in bed: TST), sleep latency (Time from going to bed to falling asleep: $\mathrm{SL}$ ), wake after sleep (The sum of the waking hours from falling asleep to waking up: WASO), and sleep efficiency (Ratio of total sleep time to total bed time: SE). If the sleep efficiency is over $85 \%$, it can be called good sleep.

\section{Analytical method}

All data were expressed as mean \pm standard deviation (SD). The relationships between sedentary behavior behavior and physical activity indices and insomnia symptom indices and between sedentary behavior behavior and physical activity indices were analyzed using Pearson's correlation coefficients. The significance level was set at less than $5 \%$.

\section{lii. Results}

\section{Audience Summary}

Of 31 participants who provided informed consent to participate in this study, 20 who had no missing data were included in the analysis. Basic information on the subjects is presented in Table 1. The mean age of the subjects was 59.0. + - 9.8 years, 12 men (60\%), 8 women, 16 inpatients ( $80 \%)$, and 4 outpatients. The mean value of chlorpromazine (CP) equivalent was $896.67 \pm 375.89 \mathrm{mg} /$ day, and the prescription was not changed in any subjects during the measurement period.

\section{Sedentary behavior Behavior and Physical Activity Index and Insomnia Symptom Index}

The mean values of the Sedentary behavior Behavior and Physical Activity Index and Insomnia Symptom Index for 1 week in 20 subjects were as shown in Table 2. Sedentary behavior behavior (Behavior below 1.5 METs during wakefulness) accounted for $73 \%$ of the awake time, and MVPA for $4.9 \%$. In addition, 8 patients had MVPA $<30$ min (inactive), and the activity of patients with schizophrenia was generally low. There was the significant and positive relation on total sedentary behavior time with bed time and sleep latency. MVPA showed a significant positive correlation with sleep efficiency and a significant negative correlation with wake after sleep onset. A significant negative correlation was found between the number of sedentary behavior bout and sleep efficiency, and a positive correlation was found between the number of sedentary behavior bout and wake after sleep. Furthermore, low-intensity physical activity was 
significantly negatively associated with bed time, total sleep time, and sleep latency, respectively (Table3).

\section{Sedentary behavior Behavior and Physical Activity Index}

The total sedentary behavior time showed a significant positive correlation with the sedentary behavior bout number and a negative correlation with the amount of low intensity physical activity. Furthermore, the sedentary behavior bout number was significantly negatively associated with MVPA and step number, respectively (Table4).

\section{CP-Converted Values and Sedentary behavior Behavior and Physical Activity Index/Insomnia Symptom Index}

There was no significant correlation between the CP-equivalent value and the sedentary behavior behavior and physical activity index (Total sedentary behavior time and sedentary behavior bout number, low intensity physical activity, MVPA) or the insomnia symptom index (Total bedtime, total sleep time, sleep latency, wake during sleep, and sleep efficiency).

\section{Discussion}

The purpose of this study was to clarify the relationship between insomnia symptoms and sedentary behavior behavior and physical activity in patients with chronic schizophrenia. The total sedentary behavior time objectively evaluated in this study showed a significant positive association between bedtime and sleep latency. There was a significant negative correlation between the number of sedentary behavior bout and sleep efficiency, and a positive correlation between the number of sedentary behavior bout and wake after sleep. In the correlation between physical activity index and sleep index, MVPA and increase in the number of steps were related to good sleep, while increase in light physical activity (LPA) was related to deteriorated sleep index. These results suggest that daytime sedentary behavior in schizophrenic patients is associated with insomnia symptoms and that a decrease in sedentary behavior may lead to an improvement in insomnia symptoms, and that physical activity, especially more than moderate intensity activity, may improve sleep quality.

The average total sedentary behavior time of the subjects in this study was very high, $11.6 \mathrm{~h} / \mathrm{day}$. A study comparing the sedentary behavior behavior of 76 schizophrenic patients with that of 38 age-, sex-, and BMI-matched normal controls (Vancampfort et al., 2012) showed that the total sedentary behavior time ( $8.5 \mathrm{~h}$ vs $6.2 \mathrm{~h}$ ) of schizophrenics was significantly longer. In addition, a previous study that objectively evaluated total sedentary behavior time in hospitalized adolescent patients with schizophrenia showed an increase in total sedentary behavior time in the latter half of adolescence (Mean $8.5 \mathrm{~h} /$ day: Vancampfort et al., 2012) at an average age of 37.0 years compared with the first half of adolescence (Mean 6.9 h/day - Strassnig et al., 2012) at an average age of 25.1 years. And, it was average $9.7 \mathrm{~h} /$ day (Stubbs et al., 2017a) in integration incontinence patient of 199 persons (Average age: 44.0 years) in the first half in the late prime, and the sedentary behavior time of integration incontinence patients seemed to 
be longer than the general healthy subject, and it tended to extend with the age, when the result (Average $11.6 \mathrm{~h} /$ day) in latter half of prime (Average age: 59.0 years) of this study was considered. One study of patients with mild cognitive impairment reported a higher incidence of anxiety, depression, and diabetes in addition to sleep disturbances among those seated for $8 \mathrm{~h}$ or more per day (Vancampfort et al., 2019). And, in the research which examined the relation between sedentary behavior action time and recognition function in 119 patients with schizophrenia, it was reported that the patients who sedentary behavior for long time had inferior motor reaction time and recognition processing than the patients who sedentary behavior for short time $(p<.05)$ (Stubbs et al., 2017b). In this study, too, the relation with sleep disorder such as the extension of sleep latency was shown in respect of the long total sedentary behavior time, and it was guessed that the decrease in total sedentary behavior time contributed to improvement of mental disorder including sleep disorder, reduction of body disease risk including diabetes mellitus prevention, lowering control of the recognition function, when it was considered with these previous research result.

Vancampfort et al. (2017) suggested that there may be regional differences in total sedentary behavior time. Sedentary behavior times as long as $9.7 \mathrm{~h} /$ day have been reported in North America ( $9.8 \mathrm{~h} /$ day), South America ( $9.3 \mathrm{~h} /$ day), and Asia (Vancampfort et al., 2017), for example, compared with European regions (Average $6.9 \mathrm{~h} /$ day). However, even when compared with these study results, the total sedentary behavior time in this study was very long, $11.6 \mathrm{~h} /$ day. The long total sedentary behavior time shown in this study may reflect the characteristics of psychiatric care in Japan. In Japan, hospitalization number of the integration incontinence patient and dosage of the antipsychotic drug are also more than foreign countries. Actually, the average of CP conversion value of the subject in this study was high with 896.67 $\mathrm{mg} /$ day, and the subject who exceeded $1,000 \mathrm{mg} /$ day which is said to be a large dose of the antipsychotic drug in the schizophrenia pharmacotherapy guideline also included 8 persons. Compared with previous studies in which the CP-equivalent value and sedentary behavior time were shown (Vancampfort et al., 2012), the CP-equivalent value and sedentary behavior time in this study were 1.3 1.4 times higher.

In this study, the sedentary behavior bout number, i.e., the number of interruptions in sedentary behavior for more than $5 \mathrm{~min}$, showed a positive association with WASO, a sleep indicator, and a negative association with SE. In general, because longer total sedentary behavior time increases the risk of health hazards (Stubbs et al., 2016; Stubbs et al., 2017a; Ellingson et al., 2018; Vancomprort et al., 2019), an increase in the number of sedentary behavior bouts that breaks sedentary behavior can be considered a favorable situation. In fact, it has been reported that the higher the sedentary behavior bout number, the lower the waist circumference and body mass index, and the better the triglyceride value as an index of neutral fat and the glucose value as an index of diabetes in healthy people regardless of the total sedentary behavior time (Owen et al., 2010). However, in this study, the more the sedentary behavior bout number was, the more the negative effect was caused for the sleep. The mean sedentary behavior bout number in this study was 121.7 time/day, which was also higher than in previous studies (43.8 92.1 time/day : Diaz, et al . , 2017), but this higher number of sedentary behavior time interruptions may represent restless symptoms of schizophrenia. In fact, the mean value of LPA from 1.5 METs to 3.0 METs 
in the subjects of this study was $204.1 \mathrm{mim} /$ day, accounting for $13 \%$ of daily physical activity, which is similar to the results of recent previous studies (Engh et al., 2019 Snethen et al., 2014). The results of this study, in which the total sedentary behavior time and the sedentary behavior bout number were larger than those of previous studies, while the LPA was almost the same, seemed to reflect the symptoms of restless schizophrenia.

In this study, we found a significant association between the level of physical activity intensity and sleep indices, in which high LPA deteriorated sleep indices, and high MVPA, representing physical activity of 3 Mets or more, improved sleep indices. The percentages of LPA and MVPA in daily physical activity in this study were $13 \%$ and $5 \%$, respectively, which were both lower than those in previous studies in general healthy subjects (LPA: 15.3\%, MVPA: 8.8\%; Kruisdijk et al., 2017). A study examining factors associated with physical activity in schizophrenic patients reported that low physical activity was associated with sleep disturbance and limitation as well as aging (Stubbs et al., 2018), and stated that failure to meet international physical activity recommendations increased the likelihood of sleep disturbance independent of depression and anxiety (Vancomprort et al., 2018). The WHO Guidelines on Physical Activity and Sedentary Behavior recommend that for adults aged $18 \sim 64$ years, moderate-intensity aerobic exercise for 150 to $300 \mathrm{~min} / \mathrm{wk}$, or high-intensity aerobic exercise for 75 to $150 \mathrm{~min}$, or a combination of both for equivalent time and intensity exercise, and 2-day/wk strength training with moderate-intensity or greater stress should be included for physical activity; sedentary behavior should be kept to a minimum, and low-intensity exercise should be substituted. In qualitative research interviewing schizophrenic patients by Faulkner and Bee (2017), it is shown that the sleep problem which patients want to improve the most is WASO. In this study, WASO was positively correlated with MVPA and step count, suggesting that MVPA and walking are effective in reducing sleep onset, one of the insomnia symptoms in schizophrenic patients. Takenouchi et al. (2019) reported in their study of inpatients with chronic schizophrenia in Japan that the higher the number of steps taken, the longer the total sleep duration and the higher the sleep efficiency. Physical activity above normal walking speed is considered MVPA, which partly supports the results of this study. And, it seemed to contribute to the sleep quality improvement of chronic integration incontinence hospitalized patient by replacing sedentary behavior time and LPA with MVPA, since the life hour in a day is limited.

In the symptom of integration incontinence, there are positive symptoms such as hallucination, delusion and annihilation thought and negative symptoms such as flattening of the feeling and hypobulia, etc., and the negative symptom often shows strongly in the chronic stage. However, Taliercio et al. (2020) reported that decreased physical activity in patients with schizophrenia was not associated with the severity of symptoms, suggesting that it is feasible to increase physical activity to enhance sleep quality in patients with chronic schizophrenia. In a study that incorporated physical therapy to promote physical activity in patients with schizophrenia and affective disorder (Gyllensten et al., 2020), it was reported that patients with affective disorder were able to reach recommended levels of moderate physical activity according to the World Health Organization guidelines, while those with schizophrenia showed a positive attitude but showed no change in behavior. It was considered that the support which leads to the behavior change, for example, that the purpose which increases the physical activity is individually set, 
and that it stimulates the action change in which MVPA increases in usual walking and stair ascent and descent, etc. in the activity of daily living, when the sedentary behavior action is interrupted, leads to the security of arousal hour in the middle and collective sleep time, and that it can improve insomnia symptom of the integration incontinence patient.

\section{囚. Limitation}

In this study, though the relation has not been recognized in CP conversion value and sedentary behavior position action index, antipsychotic drug dose seemed to affect the total sedentary behavior time in some ways, and it seemed to be future examination subject.

\section{Abbreviations}

LPA Low-intensity physical activity

MVPA CHECK TEXT / EXISTING GLOSSARY FOR DEFINITION

SE Sleep efficiency

SL Sleep latency

TIB Time in bed

TST Total sleep time

WASO Waking after sleep onset

\section{Declarations}

\section{Ethics approval and consent to participate}

This study was conducted with the approval of the Research Ethics Committee of the Research Institute of Nursing Care for People and Community at the College of Nursing Art and Science, University of Hyogo and the Ethics Review Boards of participating institutions and conducted according to the Declaration of Helsinki and subsequent revisions. Candidates for the study were those who satisfied the four conditions mentioned and who were judged by their physicians to be able to make the decision to participate in the study of their own free will. We thoroughly explained to the candidates, verbally and with documents, the purpose and significance of the study, the methods that would be employed, showing them the actual monitoring equipment to help them visualize the efforts they would be required to make to collect data. We also explained about matters such as the protection of personal information, the viewing of patients' medical records by investigators, the announcement of the study results to the public, and how data would be disposed of after the completion of the study. Only those who gave their written consent were selected as subjects for the study. If a deterioration in the mental state or physical condition of the patient 
occurred by participating in the study, monitoring was immediately stopped. We also established a system whereby subjects could contact the lead physician or a nurse at any time as required to have a medical consultation.

\section{Consent for publication}

Not applicable.

\section{Availability of data and materials}

The datasets during and/or analysed during the current study available from the corresponding author on reasonable request.

\section{Competing interests}

The authors declare that they have no competing interests.

\section{Funding}

This work was supported by JSPS KAKENHI Grant Number JP18K17529.

\section{Authors' contributions}

R. Takenouchi, M. Shibata, I. Kishida and M. Kawada designed the study. R. Takenouchi, M. Shibata, I. Kishida, C. Ishii and N. Ishii acquired the data. R. Takenouchi, M. Shibata I. Kishida and M. Kawada conducted the statistical analysis. R. Takenouchi and M. Shibata, I. Kishida, M. Kawada and M. Buyo managed the literature searches and prepared the manuscript. M. Shibata and M. Kawada and M. Buyo provided feedback about data analysis and interpretation. All authors have approved the final manuscript.

\section{Acknowledgements}

We thank the participants for their cooperation in this study.

\section{References}

1. Bueno-Antequera, J., Oviedo-Caro, MÁ., Munguía-Izquierdo, D.(2018). Relationship between objectively measured sedentary behavior and health outcomes in schizophrenia patients: The PsychiActive project. Schizophr Res, 197, 87-92. doi: 10.1016/j.schres.2017.11.022.

2. Chouinard, S., Poulin, J., Stip, E., Godbout, R.(2004). Sleep in untreated patients with schizophrenia: a meta-analysis. Schizophr Bull, 30(4), 957-967. doi: 10.1093/oxfordjournals.schbul.a007145.

3. Cohrs, S. (2008). Sleep disturbances in patients with schizophrenia : impact and effect of antipsychotics.CNS Drugs, 22(11), 939-62. doi: 10.2165/00023210-200822110-00004. 
4. Diaz, KM., Howard, VJ., Hutto, B., Colabianchi, N., Vena, JE., Safford, MM., Blair, SN., Hooker, SP. (2017). Patterns of Sedentary Behavior and Mortality in U.S. Middle-Aged and Older Adults: A National Cohort Study. Ann Intern Med, 167(7), 465-475. doi: 10.7326/M17-0212.

5. Ellingson, LD., Meyer, JD., Shook, RP., Dixon, PM., Hand, GA., Wirth, MD., Paluch, AE., Burgess, S., Hebert, JR., Blair, SN. (2018). Changes in sedentary time are associated with changes in mental wellbeing over 1 year in young adults. Prev Med Rep, 11, 274-281. doi: 10.1016/j.pmedr.2018.07.013.

6. Engh, JA., Egeland, J., Andreassen, OA., Bang-Kittilsen, G., Bigseth, TT., Holmen, TL., Martinsen, EW., Mordal, J., Andersen, E.(2019). Objectively Assessed Daily Steps-Not Light Intensity Physical Activity, Moderate-to-Vigorous Physical Activity and Sedentary Time-Is Associated With Cardiorespiratory Fitness in Patients With Schizophrenia. Front Psychiatry, 10, 82. doi: 10.3389/fpsyt.2019.00082.

7. Faulkner, S, Bee, P.(2017): Experiences, perspectives and priorities of people with schizophrenia spectrum disorders regarding sleep disturbance and its treatment: a qualitative study. BMC Psychiatry, 17(1):158. doi: 10.1186/s12888-017-1329-8.

8. Gyllensten, AL., Ovesson, MN., Hedlund, L., Ambrus, L., Tornberg, Å. (2020). To increase physical activity in sedentary patients with affective - or schizophrenia spectrum disorders - a clinical study of adjuvant physical therapy in mental health.Nord J Psychiatry, 74(1), 73-82. doi: 1 0.1080/08039488.2019.1669706.

9. Kluge, M., Schacht, A., Himmerich, H., Rummel-Kluge, C., Wehmeier, PM., Dalal, M., Hinze-Selch, D., Kraus, T., Dittmann, RW., Pollmächer, T., Schuld, A.(2014): Olanzapine and clozapine differently affect sleep in patients with schizophrenia: results from a double-blind, polysomnographic study and review of the literature. Schizophr Res, 152(1), 255-260. doi: 10.1016/j.schres.2013.11.009.

10. Kruisdijk, F., Deenik, J., Tenback, D., Tak, E., Beekman, AJ., van Harten, P., Hopman-Rock, M., Hendriksen, I.(2017). Accelerometer-measured sedentary behaviour and physical activity of inpatients with severe mental illness. Psychiatry Res, 254, 67-74. doi:

10.1016/j.psychres.2017.04.035. Epub 2017 Apr 22.

11. Laskemoen, JF., Simonsen, C., Büchmann, C., Barrett, EA., Bjella, T., Lagerberg, TV., Vedal, TJ., Andreassen, OA., Melle, I., Aas, M.(2019). Sleep disturbances in schizophrenia spectrum and bipolar disorders - a transdiagnostic perspective. Compr Psychiatry; 91, 6-12. doi: 10.1016/j.comppsych.2019.02.006.

12. Lederman, O., Ward, PB., Firth, J., Maloney, C., Carney, R., Vancampfort, D., Stubbs, B., Kalucy, M., Rosenbaum, S.(2019):Does exercise improve sleep quality in individuals with mental illness? A systematic review and meta-analysis. J Psychiatr Res.,109, 96-106. doi:

10.1016/j.jpsychires.2018.11.004.

13. Ministry of Health, Labour and Welfare.(2020). Summary of Static/Dynamic Survey of Medical Institutions and Hospital Report,2019. https://www.mhlw.go.jp/toukei/saikin/hw/iryosd/19/( 2021/03/21)

14. OECD(2020): Health Statistics2020; Health Care Utilisation : Hospital average length of stay by diagnostic categories.https://stats.oecd.org/Index.aspx?ThemeTreeld=9( 2021/03/21) 
15. Owen N, Healy GN, Matthews CE, Dunstan DW. (2010). Too much sedentary behavior: the population health science of sedentary behavior.Exerc Sport Sci Rev, 38(3), 105-113. doi:

10.1097/JES.0b013e3181e373a2. Sedentary Behaviour Research Network: What is Sedentary Behaviour? https://www.sedentarybehaviour.org/what-is-sedentary-behaviour/(2019/08/30)

16. Snethen, GA., McCormick, BP., Lysaker, PH. (2014). Physical activity and psychiatric symptoms in adults with schizophrenia spectrum disorders. J Nerv Ment Dis, 202(12), 845-852.doi:

10.1097/NMD.0000000000000216.I

17. Statistics Bureau of Japan. (2021). Population estimation. ; Elderly people in Japan from the viewpoint of statistics: February 2021 report. http://www.stat.go.jp/data/jinsui/pdf/202102.pdf. $(2021 / 3 / 28)$

18. Strassnig, M., Brar, JS., Ganguli, R. (2012). Health-related quality of life, adiposity, and sedentary behavior in patients with early schizophrenia: preliminary study. Diabetes Metab Syndr Obes, 5, 389394. doi: 10.2147/DMSO.S33619.

19. Stubbs, B., Williams, J., Gaughran, F., Craig, T. (2016). How sedentary are people with psychosis? A systematic review and meta-analysis.Schizophr Res, 171(1-3), 103-109. doi:

10.1016/j.schres.2016.01.034.

20. Stubbs B, Chen LJ, Chung MS, Ku PW.(2017a). Physical activity ameliorates the association between sedentary behavior and cardiometabolic risk among inpatients with schizophrenia: A comparison versus controls using accelerometry. Compr Psychiatry, 74, 144-150. doi:

10.1016/j.comppsych.2017.01.010.

21. Stubbs, B., Ku, PW., Chung, MS., Chen, LJ. (2017b).Relationship Between Objectively Measured Sedentary Behavior and Cognitive Performance in Patients With Schizophrenia Vs Controls. Schizophr Bull, 43(3), 566-574. doi: 10.1093/schbul/sbw126.

22. Stubbs B, Vancampfort D, Firth J, Hallgren M, Schuch F, Veronese N, Solmi M, Gaughran F, Kahl KG, Rosenbaum S, Ward PB, Carvalho AF, Koyanagi A. (2018). Physical activity correlates among people with psychosis: Data from 47 low- and middle-income countries. Schizophrenia Research, 193, 412417. doi: 10.1016/j.schres.2017.06.025.

23. Takenouchi R, Kawada M., Shibata, M.(2019). Relation between Physical Activity and Sleep Quality Data in Schizophrenic Inpatients. J Jpn Acad Nurs Sci., 39,68-73. doi: 10.5630/jans.39.68.

24. Taliercio, J., Bonasera, B., Portillo, C., Ramjas, E., Serper, M. (2020). Physical Activity, Sleep-related Behaviors and Severity of Symptoms in Schizophrenia. Psychiatry Research, 294, 113489.

25. Vancampfort D, Probst M, Knapen J, Carraro A, De Hert M.(2012): Associations between sedentary behavior and metabolic parameters in patients with schizophrenia. Psychiatry Research,200, 73-78. doi: 10.1016/j.psychres.2012.03.046.

26. Vancampfort D, Firth J, Schuch FB, Rosenbaum S, Mugisha J, Hallgren M, Probst M, Ward PB, Gaughran F, De Hert M, Carvalho AF, Stubbs B. (2017). Sedentary behavior and physical activity levels in people with schizophrenia, bipolar disorder and major depressive disorder:a global systematic review and meta-analysis. World Psychiatry, 16, 308-315. doi: 10.1002/wps.20458. 
27. Vancampfort D, Stubbs B, Smith L, Hallgren M, Firth J, Herring MP, Probst M, Koyanagi A. (2018). Physical activity and sleep problems in 38 low- and middle-income countries. Sleep Med, 48, 140147. doi: 10.1016/j.sleep.2018.04.013.

28. Vancampfort D, Stubbs B, Lara E, Vandenbulcke M, Swinnen N, Koyanagi A.(2019). Correlates of sedentary behavior in middle-aged and old age people with mild cognitive impairment: a multinational study.Int Psychogeriatr, 31(4), 579-589. doi: 10.1017/S1041610218001163.

\section{Tables}

Table1. Patient characteristics.

\begin{tabular}{ll} 
Variables & Value, \% or mean (SD) \\
\hline Age, years & $59.0(9.8)$ \\
\hline Height, $\mathrm{cm}$ & $163.7(9.5)$ \\
\hline Weight, kg & $63.0(12.1)$ \\
\hline Chlorpromazine equivalent (mg/day) & $896.67(375.89)$ \\
\hline Sex (\%) & \\
\hline Male, $\mathrm{n}$ & 12,60 \\
\hline Female, $\mathrm{n}$ & 8,40 \\
\hline Patients (\%) & \\
\hline Inpatients, $\mathrm{n}$ & 16,80 \\
\hline Outpatients, $\mathrm{n}$ & 4,20
\end{tabular}

Table 2. Mean and SD of objective sleep and activity indices. 


\begin{tabular}{ll} 
Outcome scale & Mean (SD) \\
\hline Objective sleep index & \\
\hline Time in bed (h:m) & $8: 15(1: 01)$ \\
\hline Total sleep time (h:m) & $6: 58(0: 47)$ \\
\hline Sleep latency (min) & $7.7(5.9)$ \\
\hline Waking after sleep onset (min) & $46.3(27.3)$ \\
\hline Sleep efficiency (\%) & $85.0(6.0)$ \\
\hline Activity index & \\
\hline Total sedentary time (min) & $695.9(104.3)$ \\
\hline Total sedentary bout (time) & $121.7(42.6)$ \\
\hline Light physical activity (min) & $204.1(118.6)$ \\
\hline MVPA (min) & $46(41)$ \\
\hline Steps (steps) & $5,755(2,674)$
\end{tabular}

Table 3. Correlation between Sedentary Behavior/Physical Activity Index/Insomnia Symptom Index

\begin{tabular}{llllll} 
& TIB & TST & SL & WASO & SE \\
\hline Total & $.449 *$ & .320 & $.543^{*}$ & .331 & -.274
\end{tabular}

Sedentary time

\begin{tabular}{llllll}
\hline Total & .142 & -.207 & .376 & $.503^{\star}$ & $-.592^{\star}$ \\
sedentary bout & & & & & \\
\hline Light & $-.722^{\star *}$ & $-.642^{\star}$ & $-.661^{\star}$ & -.221 & .244
\end{tabular}

Physical activity

\begin{tabular}{lccccc}
\hline MVPA & -.200 & .115 & -.287 & $-.577^{*}$ & $.536 *$ \\
Steps & .076 & .337 & -.205 & $-.461^{*}$ & .389
\end{tabular}

TIB=Time in bed, TST=Total sleep time, SL=Sleep latency

WASO=Wake after sleep onset, SE=Sleep efficiency

$n=20$

${ }^{*} \mathrm{p}<.05$ 
$\star *<.001$

Table 4. Correlation between sedentary behavior and physical activity indices

\begin{tabular}{llllll} 
& Total & Total & LPA & MVPA & Steps \\
& Sedentary time & Sedentary bout & & & \\
Total & - & $.668^{*}$ & $-.887^{* *}$ & -.217 & -.277 \\
Sedentary time & & & & & \\
\hline Total & $.668^{*}$ & - & -.402 & $-.551^{\star}$ & $-.618^{*}$ \\
Sedentary bout & & & & & \\
\hline LPA & $-.887 * *$ & -.402 & - & -.043 & -.091 \\
\hline MVPA & -.217 & $-.551^{*}$ & -.043 & - & $.896^{* *}$ \\
\hline Steps & -.277 & $-.618^{*}$ & -.091 & $.896 * *$ & -
\end{tabular}

LPA= Light-intensity physical activity, MVPA= Moderate-to-vigorous physical activity

$n=20$

${ }^{*} \mathrm{p}<.05$

$\star *<.001$ 